SCIENTIFIC REPORT

\title{
Automated identification of diabetic retinal exudates in digital colour images
}

\author{
A Osareh, M Mirmehdi, B Thomas, R Markham
}

Br J Ophthalmol 2003;87:1220-1223

\begin{abstract}
Aim: To identify retinal exudates automatically from colour retinal images.

Methods: The colour retinal images were segmented using fuzzy C-means clustering following some key preprocessing steps. To classify the segmented regions into exudates and non-exudates, an artificial neural network classifier was investigated.

Results: The proposed system can achieve a diagnostic accuracy with $95.0 \%$ sensitivity and $88.9 \%$ specificity for the identification of images containing any evidence of retinopathy, where the trade off between sensitivity and specificity was appropriately balanced for this particular problem. Furthermore, it demonstrates $93.0 \%$ sensitivity and $94.1 \%$ specificity in terms of exudate based classification. Conclusions: This study indicates that automated evaluation of digital retinal images could be used to screen for exudative diabetic retinopathy.
\end{abstract}

ntraretinal fatty (hard) exudates are a visible sign of diabetic retinopathy and a marker for the presence of coexistent retinal oedema. If present in the macular area, they are a major cause of treatable visual loss in the nonproliferative forms of diabetic retinopathy. It would be useful to have an automated method of detecting exudates in digital retinal images produced from diabetic retinopathy screening programmes.

Sinthanayothin ${ }^{1}$ identified exudates in grey level images based on a recursive region growing technique. The sensitivity and specificity reported were $88.5 \%$ and $99.7 \%$; however, these measurements were based on $10 \times 10$ windows where each window was considered as an exudate or a non-exudate region. The reported sensitivity and specificity only represent an approximate accuracy of exudate recognition, because any particular $10 \times 10$ window may be only partially affected by exudates. Gardner et $a l^{2}$ used a neural network (NN) to identify the exudates in grey level images. The authors reported a sensitivity of $93.1 \%$. Again this was the result of classifying whole $20 \times 20$ regions rather than a pixel level classification. One novelty of our proposed method here is that we locate exudates at pixel resolution rather than estimate for regions. We evaluate the performance of our system applying both lesion based and image based criteria in colour retinal images.

\section{MATERIALS AND METHODS}

We used 142 colour retinal images obtained from a Canon CR6-45 non-mydriatic retinal camera with a $45^{\circ}$ field of view as our initial image dataset. This consisted of 75 images for training and testing our $\mathrm{NN}$ classifier in the exudate based classification stage. The remaining 67 colour images were employed to investigate the diagnostic accuracy of our system for identification of images containing any evidence of retinopathy. The image resolution was $760 \times 570$ at 24 bit RGB.

\section{Preprocessing}

Typically, there is wide variation in the colour of the fundus from different patients, related to race and iris colour. The first step is therefore to normalise the retinal images across the set. We selected a particular retinal image as a reference and then used histogram specification ${ }^{3}$ to modify the values of each image in the database such that its frequency histogram matched the reference image distribution (Fig IC). In the next preprocessing step, local contrast enhancement was performed to distribute the values of the pixels around the local mean to facilitate later segmentation. ${ }^{4}$ This operation was applied only on the intensity channel I of the image after it was converted from RGB to the hue-saturationintensity (HSI) colour space, so it would not affect the colour attributes of the image.

\section{Image segmentation}

Image segmentation is a process of partitioning image pixels based on one or more selected image features and in this case the selected segmentation feature is colour. The objective is to separate pixels that have distinct colours into different regions and, simultaneously, group pixels that are spatially connected and have a similar colour into the same region. Fuzzy C-means (FCM) clustering allows pixels to belong to multiple classes with varying degrees of membership. ${ }^{5}$ The segmentation approach is based on a coarse and a fine stage. The coarse stage is responsible for evaluating Gaussian smoothed histograms of each colour band of the image, in order to produce an initial classification into a number of classes and the centre for each cluster. ${ }^{6}$ In the fine stage, FCM clustering assigns any remaining unclassified pixels to the closest class based on the minimisation of an objective function.

Figure 2A illustrates an example result after this colour segmentation stage, showing the division into three classes (cf Fig 1D). Figure 2B shows the candidate exudate regions overlaid on the original image and Figure $2 \mathrm{C}$ illustrates the result of final classification after some exudate candidate regions are eliminated (details later).

\section{Feature extraction}

To classify the segmented regions into exudate or nonexudate classes we must represent them using relevant and significant features that produce the best class separability. It is worth noting that after FCM, false positive exudate candidates arise because of other pale objects including retinal light reflections, cotton wool spots and, most significantly, the optic disc. The optic disc regions were removed before classification using our automatic optic disc localisation method. ${ }^{7}$ The segmented regions are differentiated using features such as colour, size, edge strength, and texture. We experimented with a number of colour spaces 

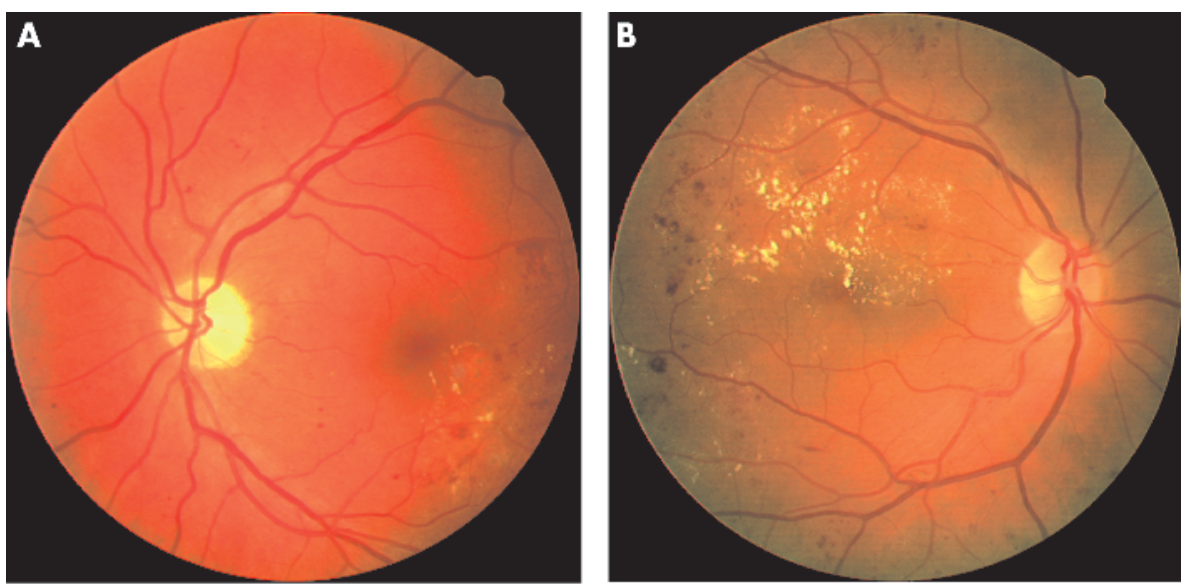

Figure 1 Colour normalisation and local contrast enhancement: (A) reference image, (B) typical retinal image (including exudates), (C) colour normalised version, (D) after contrast enhancement.
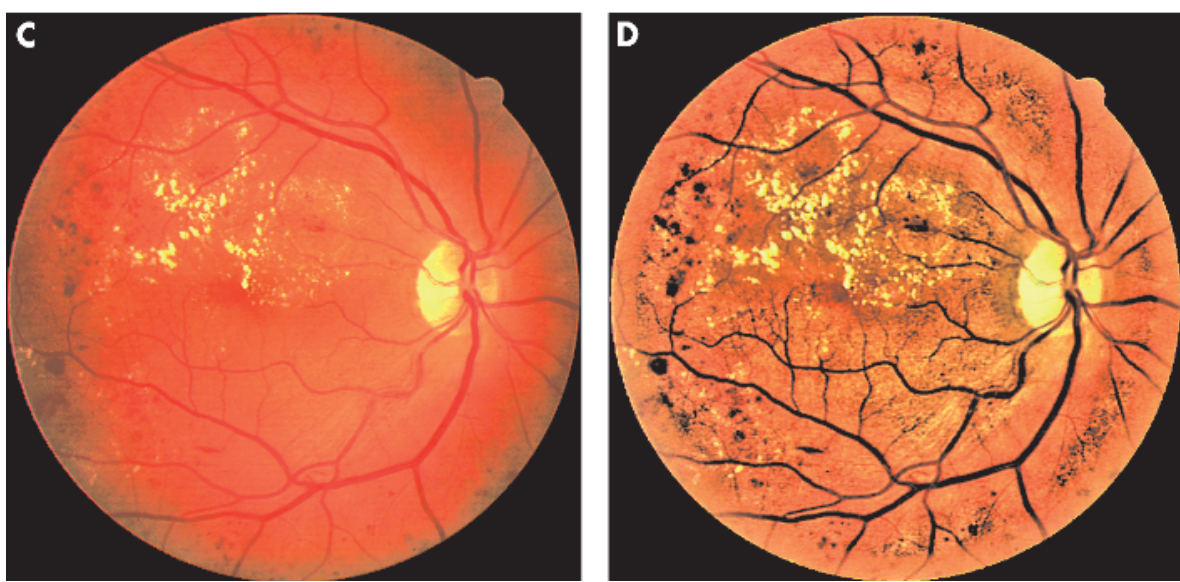

including RGB, HIS, Lab, and Luv and found that colour spaces which separate luminance and chrominance are more suitable (for example, Luv). After a comparative study of the discriminative attributes of different possible features, 18 were chosen (Table 1).

\section{Classification}

In the object classification stage we used a three layer perceptron neural network ${ }^{8}$ with 18 input nodes corresponding to our feature set. We experimented with a hidden layer with a range of 2-35 hidden units to find the optimum architecture ( 15 hidden units). A single output node gave the final classification probability. FCM clustering was applied to the 75 colour images of our prepared image dataset including 25 normal and 50 abnormal images. The outcome of the segmentation step was 3860 segmented regions consisting of 2366 exudates and 1494 non-exudates, which were considered negative cases. These regions were then labelled as exudate or non-exudate by a clinician to obtain a fully labelled dataset of examples. Two different learning methods, including standard back propagation (BP) and scaled conjugate gradient (SCG) descent ${ }^{8}$ were investigated for
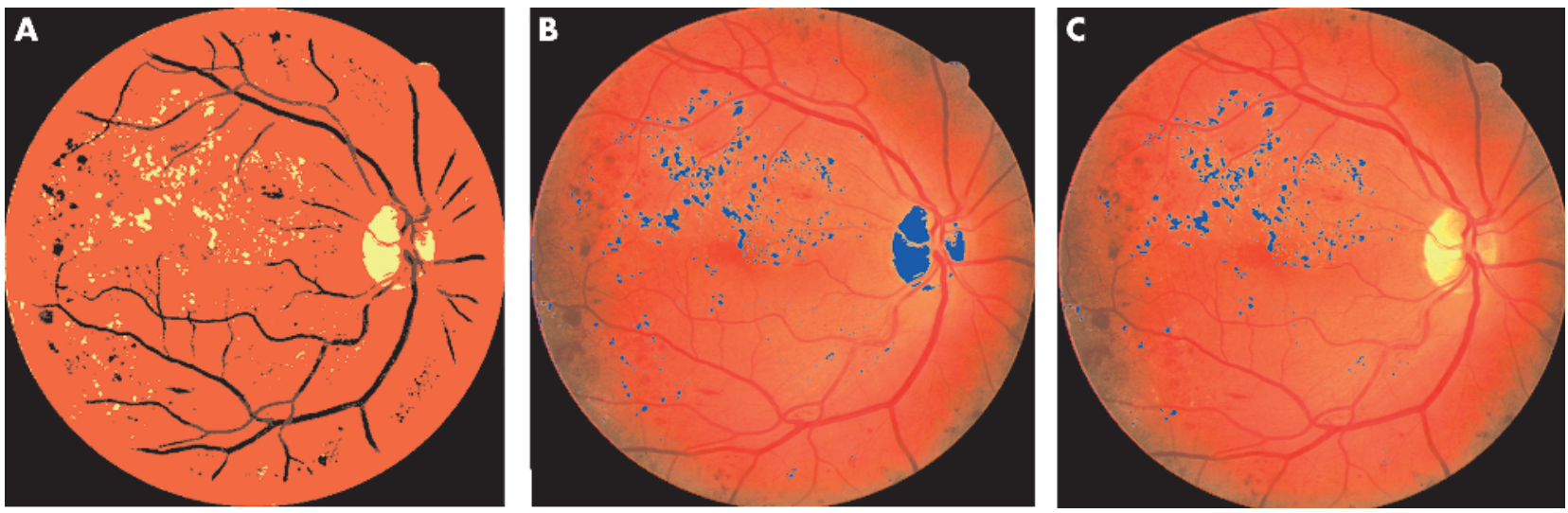

Figure 2 Colour image segmentation: (A) FCM segmented image, (B) candidate exudate regions overlaid on the original image, and (C) final classification (after subsequent neural network classification). 

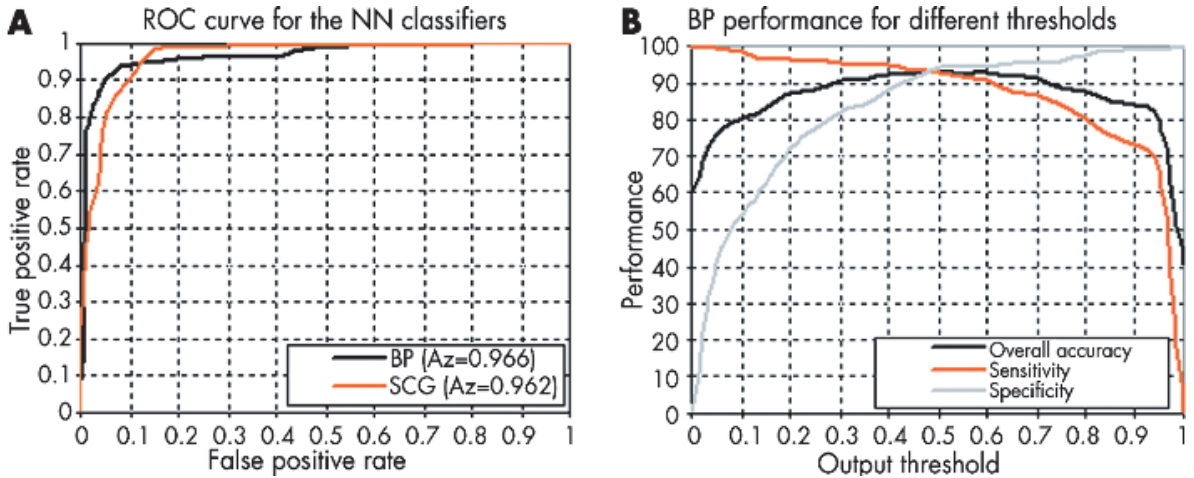

Figure 3 Performance of the BP neural network as a function of output threshold. training the NNs. Table 2 summarises the optimum results obtained on the test set for our NN configurations. For each $\mathrm{NN}$ the output threshold $(\mathrm{T})$ value giving the balance between sensitivity and specificity is also shown. The BP$\mathrm{NN}$ with 15 hidden units represented better balance between sensitivity and specificity. On the other hand, SCG could represent a higher level of sensitivity accuracy.

The optimum threshold value depends on the diagnostic requirements and can be defined based on the requirements set by medical experts. In order to demonstrate how changing this value can affect the performance of a NN classifier and the balance between sensitivity and specificity criteria, a receiver operating characteristic (ROC) curve $^{9}$ was produced. The bigger the area under the ROC curve $\left(A_{z}\right)$, the higher is the probability of making a correct decision. Figure 3A compares the behaviour of the NN classifiers for the full range of output threshold values. The BP-NN achieved a very good performance with $A_{z}=0.966$. Figure 2C illustrates the final classification result for a typical retinal image using this BP network classifier. Similarly, the SCG network with 15 hidden units, also demonstrated a high generalisation performance with $A_{z}=0.962$. Figure 3B illustrates the behaviour of the $\mathrm{BP}$ classifier and provides a guide for selecting the most appropriate output threshold based on the problem requirements and the related diagnostic accuracy including sensitivity and specificity.

So far we have discussed pixel by pixel based lesion classification. We can use this to evaluate the effectiveness of our proposed approach by assessing the image based accuracy of the system. A population of 67 different retinal images, from our initial dataset, was considered (27 normal 40 abnormal). Each retinal image was evaluated using the BP neural network classifier and a final decision was made to determine whether the image had some evidence of diabetic retinopathy. The BP classifier could identify affected retinas (including exudates) with $95.0 \%$ sensitivity while it correctly classified $88.9 \%$ of normal images-that is, the specificity.

\begin{tabular}{|c|c|}
\hline Feature & Description \\
\hline $1-3$ & Mean Lur value inside the region $\left(L \mu_{i}, U \mu_{i}, V_{\mu_{i}}\right)$ \\
\hline $4-6$ & $\begin{array}{l}\text { Standard deviation of Luv value inside the region } \\
\left(L \sigma_{i}, U \sigma_{i}, V_{\sigma_{i}}\right)\end{array}$ \\
\hline 7-9 & Mean Lur value outside the region $\left(L \mu_{0}, U_{\mu_{0}}, V_{\mu_{0}}\right)$ \\
\hline $10-12$ & $\begin{array}{l}\text { Standard deviation of Luv value outside the region } \\
\left(L \sigma_{o}, U \sigma_{o}, V \sigma_{o}\right)\end{array}$ \\
\hline $13-15$ & Luv values of region centroid $\left(L_{c}, U_{c}, V_{c}\right)$ \\
\hline & Region size (S) \\
\hline & Region compactness (C) \\
\hline 18 & Region edge strength $(E)$ \\
\hline
\end{tabular}

Table 2 Performance comparison of different classifiers (values as \%)

\begin{tabular}{llll}
\hline Classifier & & Sensitivity & Specificity \\
\hline NN-BP (15 hidden) & $(\mathrm{T}=0.50)$ & 93.0 & 94.1 \\
NN-SCG (15 hidden) & $(\mathrm{T}=0.45)$ & 97.9 & 85.2 \\
\hline
\end{tabular}

\section{RESULTS AND DISCUSSION}

We presented a study in detecting retinal exudates using FCM segmentation and a NN based on different learning methods. The best diagnostic accuracy was $93.0 \%$ sensitivity and $94.1 \%$ specificity in terms of lesion based classification, and $95.0 \%$ sensitivity and $88.9 \%$ specificity for the identification of patients with evidence of retinopathy, where the trade off between sensitivity and specificity was appropriately balanced for this particular problem.

At present, the full computation comprising segmentation, removal of false positives (for example, the optic disc), and $\mathrm{NN}$ classification takes around 11 minutes on a $700 \mathrm{MHz}$ PC which includes an unoptimised step in the optic disc removal stage $^{7}$ lasting around 10 minutes.

The results demonstrated here indicate that automated diagnosis of exudative retinopathy based on colour retinal image analysis can be very successful in detecting exudates. Hence, the system could be used to evaluate digital retinal images obtained in screening programmes for diabetic retinopathy and used by non-experts to indicate which patients require referral to an ophthalmologist for further investigation and treatment.

\section{ACKNOWLEDGEMENTS}

A Osareh is on a scholarship funded by the Iranian Ministry of Science, Research and Technology. The authors also thank the UK National Eye Research Center for their support.

\section{Authors' affiliations}

A Osareh, M Mirmehdi, B Thomas, Department of Computer Science, University of Bristol, Bristol BS8 IUB, UK

R Markham, Bristol Eye Hospital, Bristol BS1 2LX, UK

Correspondence to: Mr Alireza Osareh, Bristol University, Merchant Ventures Building, Woodland Road, Bristol BS8 IUB, UK: a.osareh@bristol.ac.uk

Accepted for publication 27 January 2003

\section{REFERENCES}

1 Sinthanayothin C. Image analysis for automatic diagnosis of diabetic retinopathy. PhD Thesis. London: King's College, 1999. 
2 Gardner GG, Keating D, Williamson TH, et al. Automatic detection of diabetic retinopathy using an artificial neural network: a screening tool. Br J Ophthalmol 1996;86:940-4.

3 Jain AK. Fundamentals of digital image processing. New York: Prentice-Hall, 1989.

4 Sinthanayothin C, Boyce J, Williamson CT. Automated localisation of the optic disc, fovea, and retinal blood vessels from digital colour fundus images. Br J Ophthalmol 1999;38:902-10.

5 Bezdek J, Keller J, Krisnapuram R, et al. Fuzzy model and algorithms for pattern recognition and image processing. London: Kluwer Academic Publishers, 1999.
6 Lim YW, Lee SU. On the colour image segmentation algorithm based on the thresholding and the fuzzy c-means techniques. Pattern Recognition 1990;23:935-52

7 Osareh A, Mirmehdi M, Thomas B, et al. Comparison of colour spaces for optic disc localisation in retinal images. In: 16th International Conference on Pattern Recognition, 2002:743-6.

8 Bishop CM. Neural networks for pattern recognition. Oxford: Clarendon Press, 1995.

9 Henderson AR. Assessing test accuracy and its clinical consequences: a primer for receiver operating characteristics curve analysis. Ann Clin Biochem 1993;30:521-39.

\section{$\mathrm{ECHO}$}

\section{Carbonic anhydrase gene 12 is overexpressed in glaucoma}

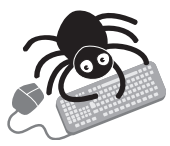

Please visit the British Journal of Ophthalmology website [wnw. bjophthalmol. com] for a link to the full text of this article. nvestigators in California and Maryland examined 16 normal (including fetal and neonatal) eyes and 10 glaucomatous eyes for the presence of various cell surface transmembrane carbonic anhydrase genes (CA). Normal developing eyes expressed CAIX and CAXII enzyme proteins in ciliary, corneal, and lens epithelia as well as the endothelium. After birth, the intensity of CAXII immunoreactivity decreased and was no longer expressed in the inner membrane of the retina. Levels fell further in adult eyes. In particular CAIX was not expressed in the ciliary epithelium although it had been very weakly expressed in developing eyes.

In glaucoma, positive immunoreactivity of both genes was weak but a striking finding was high levels of CAXII but absent CAIX expression in the non-pigmented ciliary epithelium (NPE). These patterns of expression were maintained in cultured NPE cells.

The authors conclude that CAIX and CAXII probably play an important role in aqueous humour production. Thus, the silencing of the CA9 gene in adult eyes could lead to overexpression of CA12 in NPE cells, with consequent overproduction of aqueous humour, high intraocular pressure and, hence, glaucoma.

The implications are that investigating CAl2 could provide a framework to better understand fluid equilibrium in the eye; and it might impact upon the development of more selective topical inhibitors of CAXII in treatment.

A Journal of Medical Genetics 2003;40:257-262. 